\title{
Green Synthesis and Characterization of Copper Nanoparticles Using Mixture (Zingiber officinale, Piper nigrum and Piper longum) Extract and its Antimicrobial Activity
}

\author{
RAHUL SHAH $^{1}$, AMANULLAKHAN PATHAN ${ }^{2}$, \\ HIRAL VAGHELA ${ }^{2}$, SURESH C. AMETA $^{1}$ and KOKILA PARMAR ${ }^{2}$ \\ ${ }^{1}$ Department of PG, Pacific University, Udaipur-313001 (Rajasthan), India \\ ${ }^{2}$ Department of Chemistry, Hemchandracharya North Gujarat University, Patan-384265, \\ Gujarat, India \\ amankhan255@gmail.com
}

Received 16 March 2018 / Accepted 30 March 2018

\begin{abstract}
Synthesis of copper nanoparticles using zingiber officinale, piper nigrum and piper longum extract has been studied. The synthesized copper nanoparticles were characterized using UV-Visible spectroscopy, Fourier transform infrared spectroscopy (FTIR), x-ray diffraction (XRD), field emission gun scanning electron microscopy (FEG-SEM) with EDS and high resolution transmission electron microscopy (HR-TEM). The antibacterial activity of copper nanoparticles was studied against micro organisms like against Bacillus subtilis (MTCC 441), Staphylococcus aureus (MTCC 737), Pseudomonas aeruginosa (MTCC 1681) and Escherichia coli (MTCC 1687).
\end{abstract}

Keyword: Green synthesis, Copper nanoparticles, UV-Visible, FTIR, SEM, TEM, XRD

\section{Introduction}

Nanotechnology plays a very important role in modern research, in recent years. Nanotechnology has attracted many researchers from various fields like biotechnology, physics, chemistry, material sciences, engineering and medicine. Green nanotechnology attracted many researchers from various field like physics, chemistry, material science, engineering, medicine and bio technology ${ }^{1}$. In recent, green synthesis of $\mathrm{Cu}$ nanoparticles was achieved by using microorganisms, copper nanoparticles act as antimicrobial agent in various fields. Different plants were used for the synthesis of nanoparticles using green synthesis procedure. Many researchers used green synthesis methods for different metal nanoparticles due to their growing need of eco-friendly properties.

Copper nanoparticles have wide application in medicine ${ }^{2}$. The physical and chemical methods for preparation of copper nanoparticles employ toxic chemicals ${ }^{3}$. On the other hand 
green synthesis ${ }^{4-8}$ of nanoparticles uses natural plant, fruit, leaf or seed extracts and can be safely used for medicinal applications. Generally metal nanoparticles synthesize by various methods like solution combustion, chemical displacement methods etc., ${ }^{9,10}$. Biogenic synthesis of silver nanoparticles using Crateva religiosa plant was shown good antimicrobial activity against pathogenic bacteria ${ }^{11}$. Also the green synthesis is cost impressive, environment friendly, fast in giving nanocrystalline materials. In the present work, we have found a cost effective method to synthesis copper nanoparticles using zingiber officinale, piper nigrum and piper longum extract. Synthesized copper nanoparticles were characterized using UV-Visible spectroscopy, Fourier transform infrared spectroscopy (FTIR), field gun emission scanning electron microscopy (FEG-SEM), high resolution transmission electron microscopy (HR-TEM) and x-ray diffraction (XRD).

\section{Experimental}

All the chemical reagents used in this experiment were of analytical grade purchased from ACS chemicals. The zingiber officinale, piper nigrum and piper longum powder (Figure 1) collected from around Patan (N.G.), Gujarat, India. Initially $10 \mathrm{~g}$ of powder boiled with 100 $\mathrm{mL}$ double distilled water for $30 \mathrm{~min}$ in heating on Soxhlet at temperature $60{ }^{\circ} \mathrm{C}$. The resulting product was filtered and stored in refrigerator for further use.

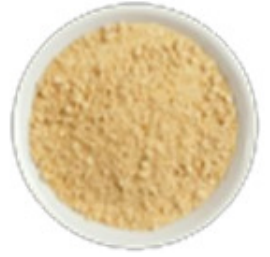

Figure 1. Mixture of zingiber officinale, piper nigrum and piper longum

\section{Syntheses of copper nanoparticles using mixture}

All solutions were prepared using double distilled water. Initially $10 \mathrm{~g}$ of powder zingiber officinale, piper nigrum and piper longum were boiled with double distilled water and heated on Soxhlet for $30 \mathrm{~min}$. then filtered it using Whatman filter paper no. 1. After filtered $20 \mathrm{~mL}$ extract was added with $30 \mathrm{~mL}$ copper sulphate. Then it is kept on magnetic stirrer with hot plate around $8 \mathrm{~h}$. The resultant solution was turned dark brown color within one hour. The solid products got it. Subsequently the solution was then centrifuged at $6000 \mathrm{rpm}$ for $20 \mathrm{~min}$. The supernatant centrifugation was used as mixture extract. Copper nanoparticles (Figure 2) were synthesized by adding $20 \mathrm{~mL}$ of mixture extract to $1 \times 10^{-3} \mathrm{M}$ copper sulphate (ACS extra pure).
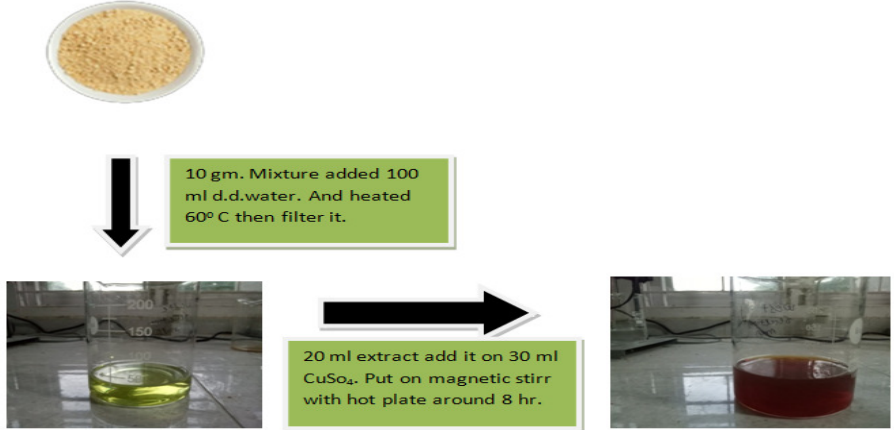

Figure 2. Green synthesis of copper nanoparticles using mixture 


\section{Characterization of copper nanoparticles}

\section{$U V$-Vis spectra analysis}

UV-Vis spectroscopy measurements (Shimadzu UV 1800) were carried out at room temperature in the region $800-200 \mathrm{~nm}$ as a function of time of the reaction. The UV-Visible absorption spectrum was used for the analysis of optical properties of green synthesized copper oxide nanoparticles. The mono dispersed copper oxide nanoparticles are shown in synthesis Figure 3. The room temperature spectra exhibited strong excitonic absorption peaks at $225-245 \mathrm{~nm}$ for samples respectively, which is in good agreement with previous work ${ }^{12}$.

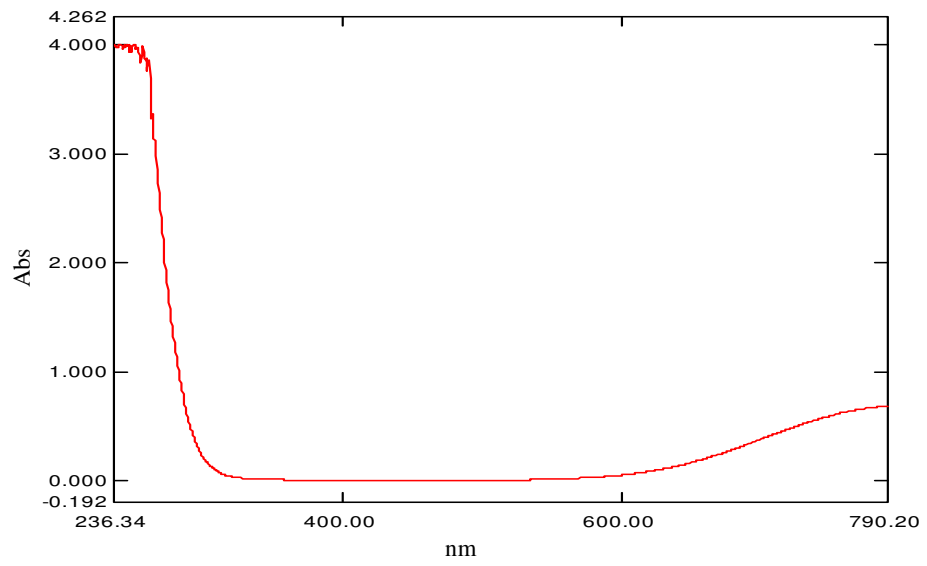

Figure 3. UV-Vis absorption spectrum of copper nanoparticles

\section{FTIR analysis of copper nanoparticles}

FTIR spectroscopy analysis was carried out to identify the biomolecules responsible for the reduction of $\mathrm{Cu}^{+}$ions. FTIR spectroscopy analysis was carried out to find the biomolecules that were bound specifically on the copper oxide nanoparticles surface. Figure 4 shows the FTIR spectra of green synthesized copper oxide nanoparticles. The spectrum showed bands at $599.86 \mathrm{~cm}^{-1}$ corresponding to metal oxygen (M-O). Strong bands were observed at 669,827 , 927 and $1354 \mathrm{~cm}^{-1}$ and have been referred to as alcohols and phenolic groups, $\mathrm{C}-\mathrm{N}$ stretching vibrations of aliphatic and aromatic amines ${ }^{13}$, respectively. The peaks around $1547 \mathrm{~cm}^{-1}$ are due to the amide bonds of enzymes or proteins ${ }^{14}$. The bands obtained at 2692 and $2879 \mathrm{~cm}^{-1}$ have been representing to stretching vibrations of primary alkanes and amines.

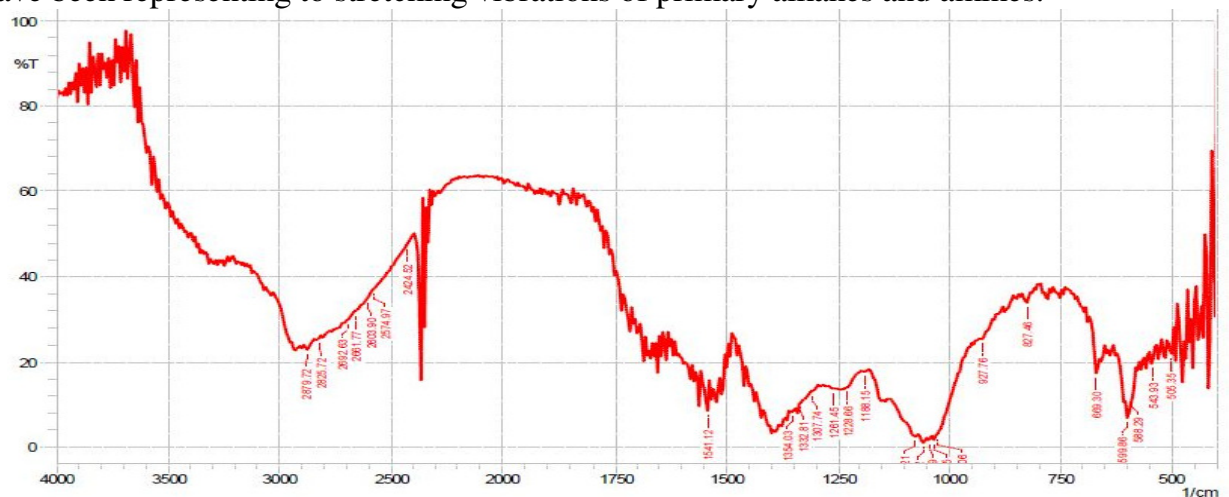

Figure 4. FTIR spectra of copper nanoparticles 


\section{$X R D$ analysis of copper nanoparticles}

X-ray diffraction (XRD) measurement of the green synthesis of copper nanoparticles carried out on a Rigaku D/max $40 \mathrm{kV}$ diffractometer equipped with the graphite mono chromator and $\mathrm{Cu}$ target. Figure 5 shows the XRD analysis of green synthesized copper oxide nanoparticles. This is used for further confirmation of copper oxide phase of nanoparticles. The observed intense peaks are $28^{0}, 32^{0}, 38^{\circ}, 44^{\circ}, 64^{\circ}$ and $77^{\circ}$ respectively representing the (100), (110), (111), (200), (210) and (220). Bragg's reflection angles based on the crystal of copper nanoparticles. These phases were indexed to spherical shape, which was compared to the data from JCPDS card No. 04-0836. The average crystallite size of the copper nanoparticles was calculated using peak broadening profile by Debye Scherer's formula as follows,

$$
\mathrm{D}=\frac{0.9 \lambda}{\beta \cos \theta}
$$

Where, $\mathrm{D}$ is average particles size, $\lambda$ is wavelength (1.5418 $\AA$ ), $\theta$ is the Bragg's angle and $\beta$ is full width half maximum (FWHM) of corresponding peek. The Scherer's formula was used to estimate the particles sizes and was found to be in the range of 15-30 nm. Gunalan et al., ${ }^{15}$ have been reported that Aloe barbadensis mediated copper oxide nanoparticles were spherical in nature and sizes ranged from $15-30 \mathrm{~nm}$.

\section{FEG-SEM and EDS analysis of copper nanoparticles}

Field emission gun scanning electron microscope (FEG-SEM) and EDS images were recorded on a JSM-7600F series instrument. FEG-SEM spectra of green synthesis of copper nanoparticles are shown in Figure 6(a-d). Copper nanoparticles by this method show nearly mono dispersed distribution of particle sizes. The average particle size of the $\mathrm{Cu}$ nanoparticles is around 15-30 $\mathrm{nm}$. The composition of copper nanoparticles was further probed by energy-dispersive x-ray (EDS) analysis. Figure 7 shows the EDS pattern of CuNPs prepared using copper sulphate, which indicates the presence of $\mathrm{Cu}$ and small amount of oxygen. EDS spectrum of copper nanoparticles shows the peaks for copper and respective elements indicating the formation of copper nanoparticles. Peak indexing of the elements is oxygen $0.5 \mathrm{keV}$ and copper 0.9 and $8.1 \mathrm{keV}$. The compositions in the mass percentage of the elements are oxygen $45.12 \%$ and copper $41.21 \%$. The experimental composition matches with the theoretically calculated composition.

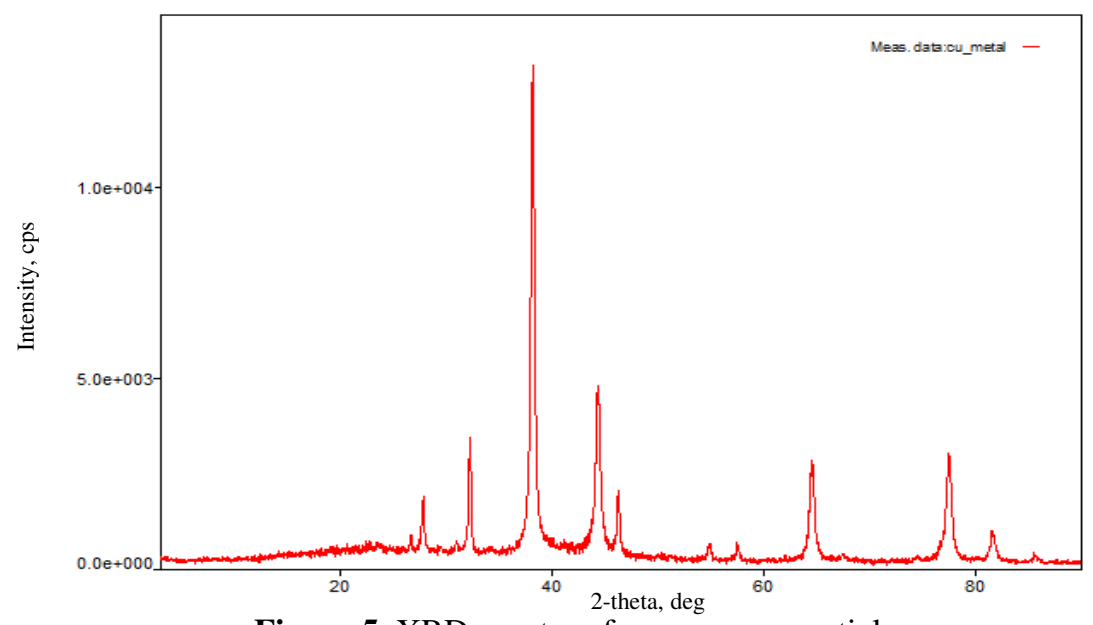

Figure 5. XRD spectra of copper nanoparticles 


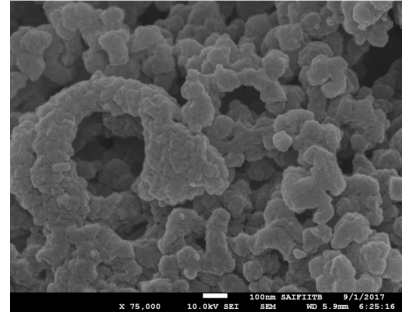

(a)

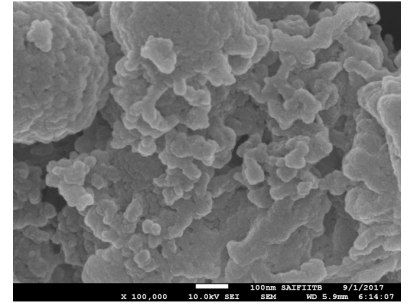

(c)

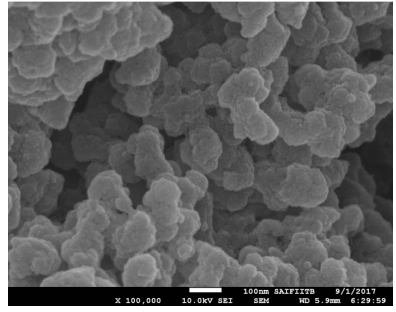

(b)

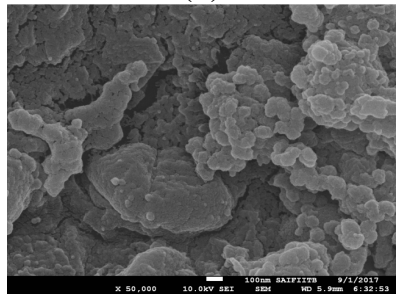

(d)

Figure 6. FEG-SEM images of copper nanoparticles

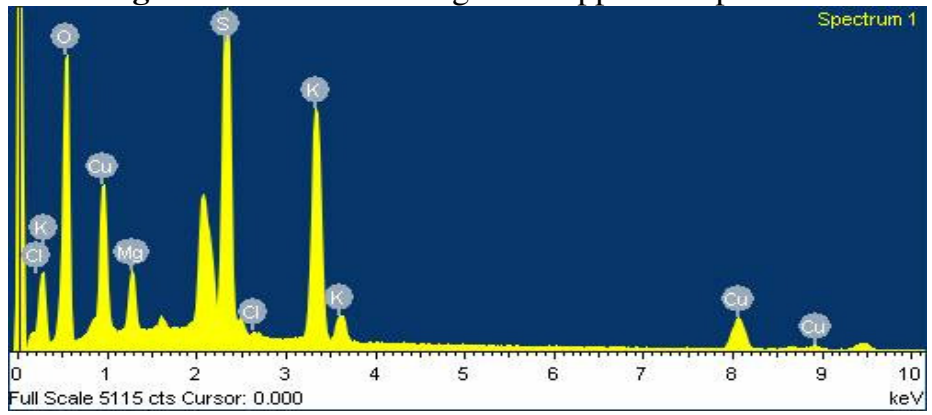

Figure 7. EDS spectra of copper nanoparticles

HR-TEM analysis of copper nanoparticles

High resolution transmission electron microscope (HR-TEM) images were recorded on a Tecnai G2-F30 electron microscope. Figure 8(a-d) shows the HR-TEM images of CuNPs prepared using mixtures. The sample preparation was carried out via the coating on carbon coated grid $\mathrm{Cu}$ Mesh 300 prior to the measurement. High resolution transmission electron microscopy (HR-TEM) has been employed to characterize the size, shape and morphology of synthesized copper nanoparticles.

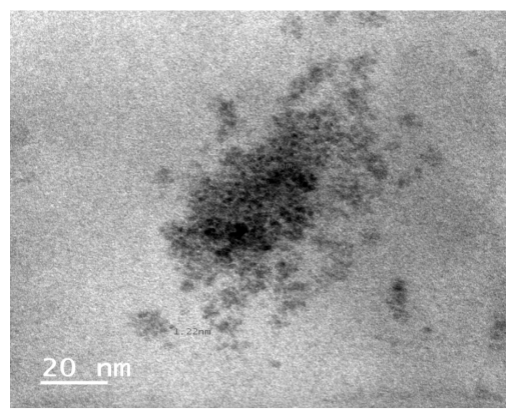

(a)

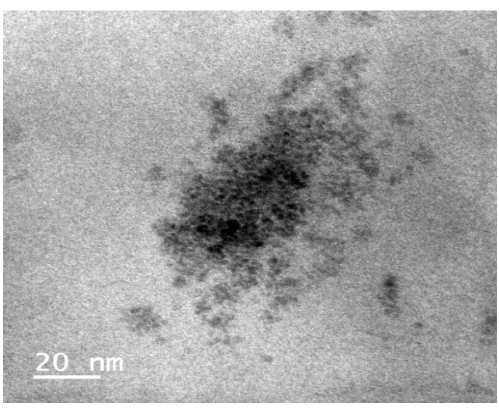

(b) 


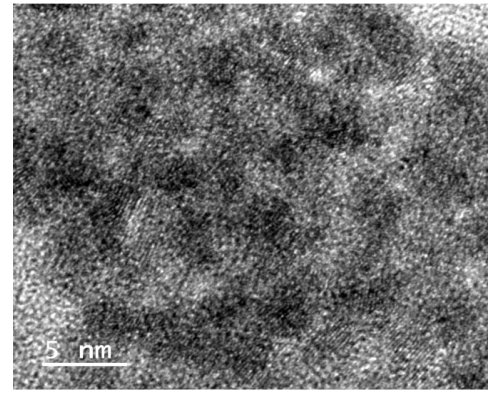

(c)

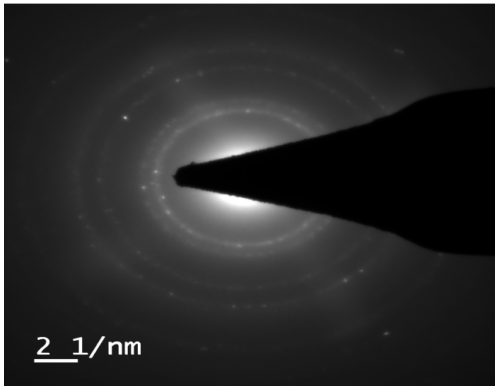

(d)

Figure 8. HR-TEM images of copper nanoparticles

\section{Antimicrobial test}

The antibacterial screening of the synthesized copper nanoparticles performed by both agar well diffusion method and paper strip method against Bacillus subtilis (MTCC 441), Staphylococcus aureus (MTCC 737), Pseudomonas aeruginosa (MTCC 1681) and Escherichia coli (MTCC 1687).

Same process applied for plant extract for comparison, fresh overnight culture of each strain swabbed uniformly by cotton on plates containing sterile Luria Bertani agar and 4 wells (diameter size- $6 \mathrm{~mm}$ ) were prepared using cup borer. $50 \mu \mathrm{L}$ of sample nanoparticles pour into each well and commercial disc of gentamicin was used as positive control. Incubated it for $24 \mathrm{~h}$ at $37{ }^{\circ} \mathrm{C}$, after that around the well (well No. 1-3 in Table 1) diameter of inhibition zone was observed in millimeter (Figure 9 and Table 1). Inhibition zone of bacterial growth is due to inhibitory compounds from the tested sample. We concluded that agar well diffusion method exhibited good antibacterial activity in which Bacillus subtilis showed excellent zone of inhibition and Escherichia coli showed weak results compare to all bacteria. Plant extract didn't give any results. Experiments with each strain performed three times for good results.

Table 1. Results of antimicrobial test

\begin{tabular}{cccc}
\hline \multirow{2}{*}{ Microorganism } & \multicolumn{3}{c}{ Zone of inhibition } \\
\cline { 2 - 4 } & $20, \mu \mathrm{L}$ & $40, \mu \mathrm{L}$ & $60, \mu \mathrm{L}$ \\
\hline B. subtilis & $2 \mathrm{~mm}$ & $8 \mathrm{~mm}$ & $11 \mathrm{~mm}$ \\
S. aureus & $1 \mathrm{~mm}$ & $6 \mathrm{~mm}$ & $12 \mathrm{~mm}$ \\
P. aeruginosa & $1 \mathrm{~mm}$ & $7 \mathrm{~mm}$ & $13 \mathrm{~mm}$ \\
E. coli & $2 \mathrm{~mm}$ & $8 \mathrm{~mm}$ & $12 \mathrm{~mm}$ \\
\hline
\end{tabular}

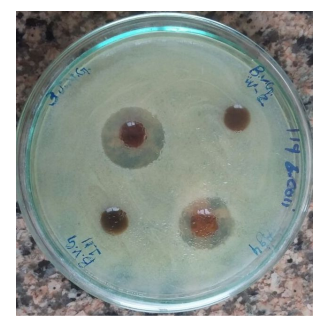

(a) B.subtilis

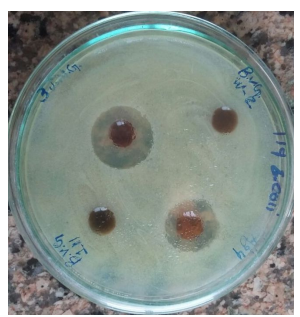

(b) S.aureus

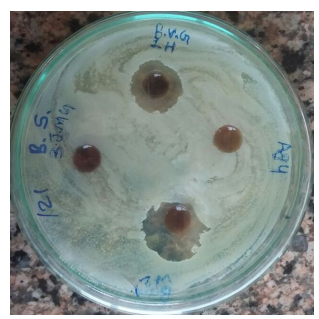

(c) P.aeruginosa

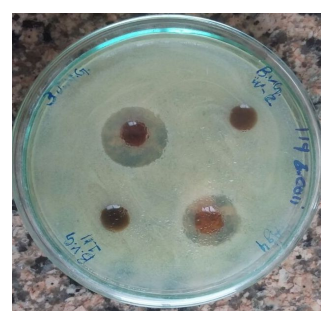

(d) E.coli

Figure 9. Antimicrobial activity of copper nanoparticles 


\section{Conclusion}

The green synthesis of copper nanoparticles performed using mixture (zingiber officinale, piper nigrum and piper longum) without involving any toxic chemicals. In this reduction reaction metal ions were reduced $\left(\mathrm{Cu}^{+2}\right.$ to $\left.\mathrm{Cu}^{+1}\right)$ very rapidly and reaction was finally completed within $8 \mathrm{~h}$ to produce copper nanoparticles. Different plants will take different time to complete the reaction due to have their different properties. The characterization of synthesized copper nanoparticles elucidated by microscopic and spectroscopic techniques which includes FEG-SEM with EDS, FTIR, HR-TEM, XRD and UV-Visible confirms the formation of copper nanoparticles. Synthesized copper nanoparticles showed high stability even after six months at ordinary room temperature. They exhibited an excellent antibacterial activity against mentioned all pathogenic bacteria. So it can concluded that biologically synthesized copper nanoparticles using mixture (zingiber officinale, piper nigrum and piper longum) can be used as environment-friendly with low cost and excellent antibacterial activity.

\section{References}

1. Vasudev D Kulkarni and Pramod S Kulkarni, Int J Cheml Stud., 2013, 1(3), 1-4.

2. Frattini A, Pellegri N, Nicastro D and De Sanctis O, Mater Chem Phys., 2005, 94(1), 148-152.

3. Schmid G (in), Schmid G, (Ed)., Nanoparticles From Theory to Application. $2^{\text {nd }}$ Ed., Wiley-VCH; Weinheim, 2010, 214-232.

4. Kaler A, Patel N and Banerjee U C, Curr Res Inf Pharm Sci., 2010, 11(4), 68-71.

5. Jagajjanani Rao K and Santanu Paria, Mate Res Bull., 2013, 48(2), 628-634; DOI:10.1016/j.materresbull.2012.11.035

6. Khan M, Khan M, Adil S F, Tahir M N, Tremel W, Alkhathlan H Z, Al- Warthan A and Siddiqui M R, Int J Nanomed., 2013, 8, 1507-1516; DOI:10.2147/IJN.S43309

7. Singh RP, Magesh S and Rakkiyappan C, Int J Bio-Eng Sci Technol, IJBEST., 2011, 2(3), 64-73.

8. Elias Saion, Elham Gharibshahi and Kazem Naghavi, Int J Mol Sci., 2013, 14(4), 7880-7896; DOI:10.3390/ijms 14047880

9. Pathan, Amanullakhan A, Kavita R Desai, Shailesh Vajapara and Bhasin C P, Adv Nanopart., 2018, 7(1), 28-35; DOI:10.4236/anp.2018.71003

10. Tejani Jayadip, Rahul Shah, Hiral Vaghela, Trupti Kukadiya and Amanullakhan A Pathan, J Nanotechnol Adv Mater., 2018, 6(1), 1-7.

11. Hiral Vaghela, Kokila A. Parmar, Amanullakhan Pathan, Kavita R Desai Jadhav Jayesh and Rahul Shah, J Appl Chem., 2016, 9(8), 29-33; DOI:10.9790/57360908012933

12. Huang J, Li Q, Sun D, Lu Y, Su Y, Yang X, Wang H, Wang Y, Shao W, He N, J. Hong J and Chen C, Nanotechnology, 2007, 18(10), 105104-105115;

DOI:10.1088/0957-4484/18/10/105104

13. Shankar S Shiv, Absar Ahmad and Murali Sastry, Biotechnol Prog., 2003, 19(6), 1627-1631; DOI:10.1021/bp034070w

14. Shah M A, Afr Rev Phys., 2008, 2(1)

15. Gunalan Sangeetha, Rajeshwari Sivaraj and Rajendran Venckatesh, Spectrochim Acta Part A: Mol Biomol Spectros., 2012, 97, 1140-1144; DOI:10.1016/j.saa.2012.07.096 